\title{
ON METOPISM
}

\author{
L. BOLK
}

Director of the Anatomical Institute, University of Amsterdam

NINE FIGURES

It is a well-known fact that in man the two frontal bones in a certain number of individuals do not coalesce. In normal eircumstances the frontal or metopical suture begins to disappear during the last quarter of the first year, and is completely closed before the end of the second year, the anterior fontanelle disappearing during the third year. The phenomenon of a persisting frontal suture generally is designed as metopism.

Many publications on metopism are contained in the anthropological and anatomical literature. Several reasons have induced me to add the present paper to them. Firstly, I am able to deal with data unknown till now regarding the numerical occurrence of the phenomenon in Dutch skulls. Such a communication is not wholly superfluous because the frequency of metopism varies not inconsiderably among different peoples or races. The second reason for the publication of this paper is given by the fact that in many points the results of my investigations contradict those of other investigators, and, as to the etiology of the phenomenon, I differ from the current opinion. Commonly an increased intracranial pressure, caused by the somewhat more strongly developing frontal brain, is regarded as the mechanical factor preventing the fusion of the two frontal bones. So Martin in his Manual of Anthropology says:

All this shows that a more considerable growth of the frontal cerebrum, as occurring in some brachycephalic groups, is to be considered the cause of metopism. By the internal pressure the normal concrescence of the frontal bones is prevented, likewise in hydrocephalic skulls, in which regularly the metopical suture persists. 
After having communicated the results of my own investigation I will enter into some critical remarks upon this opinion.

The above mentioned explanation of metopism gives rise to a more extended point of view. Some authors believe that a large brain indicates intellectual superiority. And it is easy to understand that to such a metopical suture too, should be a symptom of such a superiority, being a suture caused by a strongly developed brain. This opinion has in fact the approval of Schwalbe. In an investigation into the occurrence of a frontal suture in apes and monkeys this author, after having mentioned the current opinion with regard to the etiology of metopism, says: "This hypothesis agrees with the idea that persons with metopical crania are to be considered as being intellectually on a higher level."

The partisans of this hypothesis surely may advance the following anthropological fact, in favor of their view. It is incontestable that metopism occurs more frequently in culture races than in those possessing a lower degree of civilization. The differences are sufficiently pointed out in the following table, most of whose data are taken from Martin's Manual of Anthropology.

\begin{tabular}{|c|c|}
\hline \multicolumn{2}{|c|}{ Frequency of metopism } \\
\hline & \\
\hline Australian. & 1.0 \\
\hline Negroes.... & 1.2 \\
\hline Malayan... & 2.8 \\
\hline Papuan..... & 4.3 \\
\hline Slaves...... & 6.4 \\
\hline Alsatians... & 6.5 \\
\hline Bavarians.. & 6.4 \\
\hline Swiss....... & 7.1 \\
\hline Hamburgher & 9.5 \\
\hline Scotchman. & 9.5 \\
\hline Parisian.... & 9.7 \\
\hline
\end{tabular}

The difference between the civilized and uncivilized peopie is a very obvious one. And even when rejecting the hypothesis of any relation between metopism and intellectual development, this difference still retains its anthropological significance to the full. 
Furthermore it is clear that even among the Europeans the ratio is not at all constant in crania of the inhabitants of the MidEuropean region (Bavarians, Alsatians and Swiss), and in the Slavs the frontal bone seems to be divided less frequently than $\mathrm{n}$ crania of the inhabitants of the North-European regions (Hamburgher, Scotch, and, as will be demonstrated further on, also Amsterdamian). I draw special attention to this fact, which does not agree with the not seldom expressed contention, that metopism occurs more frequently in brachycephalic than in dolichocephalic skulls. As far as I am aware, it was Welcker who first pointed out this idea. And it is found in most treatises on metopism. But I think in most of these it is a mere statement of a current opinion, and not a result of definite investigation. The results of research do not confirm this hypothesis. This will be demonstrated by my own research in the course of this paper, and the investigations of Bryce on Scottish crania give similar results. As is well known these are very dolichocephalic, and yet the author found 9.5 per cent metopical skulls among them. Therefore among the dolichocephalic Scotchmen the metopical skulls are more numerous than is the case among the more broad-headed inhabitants of the Mid-European region. This contradicts the assumed prevalence of metopism in brachycephalic skulls.

Before finishing these introductory remarks it is necessary to give a brief account of some of the principal points in the comparative anatomy of the frontal suture. A knowledge of these points is necessary for the thorough understanding of my explanation of metopism, which, as already mentioned, differs from the current one. That the frontal bone in the human embryo arises by two points of ossification situated symmetrically is due to the fact that originally this bone was a paired one. As a rule this condition persists not only in the lower vertebrates, but even among mammals there are many groups in which the metopical suture does not disappear. In Prosimiae as a rule the frontal suture persists as long as the other sutures of the skull. In case of an early closure of the system the frontal suture also disappears early, in case of a persistence of the system till an advanced age, the frontal suture also persists. There is considerable va- 
riability as to the age at which the skull bones unite in Prosimiae. In monkeys the ossa frontalia unite and a persisting metopical suture is an individual and rare exception. Finally, in Anthropoids a metopical suture in an adult skull has never been seen.

The history of the metopical suture therefore is a somewhat complicated one. Originally the suture was always present, later it disappears, and finally in man it reappears as a not infrequent variation.

I wish to emphasize, that in consequence of this behavior of the frontal suture in the course of evolution, two possibilities must be taken into consideration when trying to account for its reappearance in man. Firstly this reappearance can be explained as due to a quite new influence acting only in man, namely the increased development of the brain which prevents the two frontal bones from uniting. But there is another point of view of a more physiological nature, claiming our full attention in no lesser degree. In primitive Primates the metopical suture persisted. In the further course of evolution certain causes, to which I intend to return, exerted their influence in such a way that both frontal bones were compelled to unite and the metopical suture disappeared. Now, I believe, the possibility presents itself that the metopical suture in man reappears, just because the factor, which once caused its disappearance in monkeys, no longer exerts its influence in the human skull. From this point of view the problem has not yet been examined.

In the foregoing it is made clear that the metopism of the human skull is the starting point of some very interesting problems, to which I will shortly refer in the order in which they are treated on the next pages. Firstly the question about the frequency of the anomaly in Dutch skulls will be discussed, then the question whether the metopical suture occurs more frequently in brachycephalic skulls, and. whether it is true that a persisting frontal suture is of some influence upon the shape of the skull. Thereupon we will examine if there exists any relation between metopism and intellectual development, particularly if it is true that the anomaly is more frequent in large skulls, containing a 
heavier brain than usual, and finally we will enter into the question of the aetiology of metopism in men.

The material I used for this research consists of 1400 adult skulls of inhabitants of Amsterdam who died during the second half of the last century. It was gathered from one of the cemeteries of this town.

In this collection I found 134 skulls with a persisting metopical suture, that is 9.5 per cent. This relation equals that found by Bryce in Scottish skulls and by Simon in Hamburghian skulls, and agrees nearly with that found by Broca among the old Parisian skulls.

As mentioned in the introductory remarks, it is often claimed in the literature that the metopical suture occurs more frequently in brachycephalic than in. dolichocephalic skulls. Now, we will examine in the first place whether this statement agrees with the results of my own research. As a dolichocephalic skull I mean in the following pages all those with an index cephalicus lower than 80 , omitting therefore a more detailed classification in mesocephalic, hyperdolichocephalic, etc.

The number of brachycephalic crania present in the whole collection of 1400 skulls, amounted to 420 , or just 30 per cent, and among the 134 metopical skulls, there were 55 or 41 per cent brachycephalic. The number of brachycephalic skulls among metopical erania surpasses, therefore, that among the collection as a whole and the difference of 11 per cent really seems to be very considerable. Only the fact merits mention that the absolute number of metopical skulls (134) is a relatively small one, and hence a few skulls more or less exert a perceptible influence upon the percentage. Altogether the above described relation proves that the majority of the metopical skulls is not brachycephalic. And therefore I do not agree with the statement of Anntchin that "metopical dolichocephalic skulls are relatively rare." This conclusion, moreover, does not agree with the results of the investigation of Bryce who, among his material of Scottish skulls, only met with two brachycephalic crania. Yet in another way the eventual influence of a persisting metopical suture upon the shape of the skull may be verified, namely in 
comparing the average index cephalicus in normal and metopical skulls. In doing so the following averages were found. That of the total number of 1400 skulls amounted to 78.3 and that of the 134 metopical skulls, 78.9. This difference is such an insignificant one that it does not prove anything as to a supposed more brachycephalic character of metopical skulls. And the average index cephalicus is such a low one that it by no means justifies the opinion that brachycephaly is a characteristic of metopical skulls, or that metopism in general is favorable to the formation of brachycephalic skulls.

Finally I wish to advance still another proof of the absence of any relation between the shape of the skull and the persistence of a frontal suture. Among the 1400 skulls there were 23 with the very low index cephalicus of 71 , an indication of a very narrow skull. And among the 134 metopical skulls, five were found with the mentioned low index. This fact demonstrates clearly that metopism occurs even frequently in skulls which are dolichocephalic in high degree.

It is well known that for the characterization of a skull its index cephalicus is a very insufficient indicator, because for instance the height of two crania with quite the same index can differ considerably, or the curvatures of the calvarium can be very dissimilar. And finally this index furnishes not a single indication as to the absolute dimensions of the skull, a very large and a very small skull may have an equal index cephalicus. Hence a comparison of this index in regard to persisting metopical sutures is a very insufficient means of recognizing the existance of an eventual relation between the shape of the skull and the frequency of metopism. It is necessary to prosecute our investigation in still another direction.

First we will examine whether the three principal dimensions of the skull in average are different in normal and metopical crania. A comparison of the sum of these averages in both groups of skulls will enable us moreover to answer the question whether it is true that metopical skulls commonly are larger, including a heavier brain than nonmetopical crania. 
In the next table the averages are dealt with of the three principal dimensions of the 134 metopical skulls and of the total number of 1400 skulls.

\begin{tabular}{|c|c|c|c|}
\hline & $\begin{array}{l}\text { AVERAGE } \\
\text { LENGTH }\end{array}$ & $\begin{array}{l}\text { AVERAGR } \\
\text { BREADTH }\end{array}$ & $\begin{array}{c}\text { AVERAGF } \\
\text { HEIGHT }\end{array}$ \\
\hline 134 metopical skulls. & 182 & 144.8 & 128.4 \\
\hline 1400 skulls. . . . . . . . . & 183.3 & 143.8 & 128.6 \\
\hline
\end{tabular}

The height of the skull was measured from the bregmapoint to the casion.

As is clearly shown by the table, the height of metopical skulls does not differ from the usual measure, for a decrease of $0.2 \mathrm{~mm}$. is of no consequence. Regarding this dimension it is certain that there exists no preponderance in metopical skulls. And also the two other dimensions scarcely testify in favor of such a supposition. For though it is true that metopical skulls average $1 \mathrm{~mm}$. broader than normal skulls, their length, on the contrary, is a somewhat smaller one. The metopical skulls seem to be shorter and broader than normal skulls. But the differences are so insignificant that the capacity of metopical skulls equals that of crania with united frontal bones. And an equality of capacity includes an equality of brain weight.

Thus it is obvious that neither in the shape, nor in the absolute dimensions is there a striking difference between the two groups of crania. In this regard the results of my investigation does not agree with that of some other authors. The metopical skulls which I examined were not more brachy-cephalic and were not larger than the normal skulls with which they were compared. And not without reason I consider the results of my own researches to be of a greater value than the contradictory results of some other investigators. For the 134 metopical skulls belonged to the same group as the non-metopical with which they were compared, the whole collection originating from one source. And this was not always the case with the material used hitherto by other investigators.

The result of my research does not harmonize with the already mentioned views upon the cause of metopism. I summarize that 
a heightened intracranial pressure during growth due to a greater development of the brain, is considered to be the cause of metopism. Now, I cannot agree with this opinion, for as clearly shown in the foregoing pages, metopism is independent of the shape as well as of the size of the skull. And if there really existed some relation between the degree of development of the brain and the frequency of metopism, one should expect among the largest skulls an increased number of metopical specimens and higher average values of the mean dimensions in metopical skulls. This is not at all the case. The averages of the three dimensions in metopical skulls are nearly the same as in the nonmetopical. Therefore a noticeable difference between the capacity of both groups of skulls cannot be accepted, and consequently the average weight of the brain must be the same.

An objection of more general theoretical nature against the current opinion about the etiology of metopism may be adduced. Is it really true that an increase of the intracranial pressure may prevent the coalescence of two bones of the skulls whose normal fate is to unite together? Martin, the renowned anthropologist, accepts this view, founding his opinion upon hydrocephalic skulls, in which, as he says, metopism is a common phenomenon.

I do not know how far this statement of the painstaking investigator is based upon observations by himself, or is merely the expression of a doctrine propagated in craniological literature. I am inclined to believe the latter. For the experience gained by myself upon this matter is in contradiction with the idea mentioned. There is no concurrence of hydrocephaly and metopism, hydrocephaly being not at all a condition propitious for the persistance of the frontal suture. I have examined carefully the hydrocephalic skulls present in the anatomical Museum of Amsterdam, and the results of this investigation are dealt with in the next table. This table informs us of the state of the frontal suture the horizontal circumference of the skull and the age. With regard to the circumference it may be remarked, that in normal Dutch skulls it amounts to $516 \mathrm{~mm}$. 


\begin{tabular}{c|c|c|c}
\hline No. & CIRCUMFERENCE & AGE & sUtURA FrontaLIs \\
\hline & $m m$. & years & \\
1 & 684 & 8 & Disappeared \\
2 & 673 & 20 & Disappeared \\
3 & 616 & 32 & Existing. Sut.sag- \\
& & & ittalis entirely \\
& & adult & closed \\
4 & 600 & adult & Disappeared \\
5 & 582 & 5 & Disappeared \\
6 & 570 & & \\
\hline
\end{tabular}

By this table it is clearly shown that the assertion that hydrocephaly regularly is accompanied by metopism, is a false one. Only in the third case the suture was still open. But it is a question whether in this case the presence of the suture was due to supposed mechanical influence of the hydrocephaly. For as mentioned in the table, in this case the sagittal suture was already entirely closed. And this fact justifies the supposition that in this case the skull was a metopical one by inheritance, in which therefore the suture also should have persisted, if the development of the brain had been quite normal. But, I admit, this to be a mere supposition, although I believe that this case may scarcely be accepted as a proof that metopism is caused by hydrocephaly. It seems better to disregard this case in a discussion of this matter. Furthermore the other data of the table afford a strong proof against the existence of such a casual relation. The first two crania are of an extraordinary size, with a circumference met with rarely, even in hydrocephalic skulls. Surely in both individuals the intracranial pressure must have been an excessive one. And notwithstanding this circumstance the frontal sutures vanished without leaving a single trace. And the same occurred in the other cases mentioned in the table.

I believe the data of this table to be sufficient to justify my statement, that hydrocephaly by no means produces, as a rule, metopism. Hence it seems to me an error to pretend that an increased intracranial pressure-caused by a marked development of the brain - is the cause of metopism. For, if the considerable increase of this pressure, as surely occurred in the skulls 
of the first two individuals of the table, was unable to prevent the coalescence of the two frontal bones, it is wholly unthinkable that a somewhat increased development of the brain will suffice to prevent these bones from uniting.

One may advance still another more weighty question with regard to the influence of the growing brain upon the skull. It is assumed that the pressure exercised by the growing brain upon the inner surface of the skull rises, when the brain is developing in a greater degree. Is this assumption true? I do not believe it. It seems to me more probable that with regard to the expansion due to their growth, the brain and the skull form one entity, the same hereditary factors determining the growth intensity of the brain as well as of the cranium. I do not believe that the dilatation of the latter is a mere mechanical phenomenon, depending on the pressure exercised by its contents. To some degree this may be the case in pathological circumstances, as in hydrocephaly or in premature closure of some suture or other, but under normal circumstances, I believe the intracranial pressure always to be the same, varying only between its physiological limits.

As a further argument in favor of the assumed influence of the growing brain upon the expansion of the skull, the fact is advanced that the forehead in metopical skulls is broader than in those with normal closure of the frontal suture, this increase of the transverse frontal measure being another result of the more strongly developing frontal lobes of the brain. Without doubt, the observation made f. i. by Welcker and Papillante is right, and I am able to confirm the same, the metopical skulls of my collection having an average breadth of the forehead of $99.7 \mathrm{~mm}$. and the nonmetopical one of $96.5 \mathrm{~mm}$. But I cannot agree with the interpretation of the phenomenon given by the above mentioned authors. I think in this matter they are confusing cause and effect. The difference may be elucidated in the following way. If the frontal suture does not disappear during the second year, the apposition of bony tissue in it is continued during a longer space of time than in case of its disappearance in the normal way, and therefore there is a very favorable 
opportunity for the forehead to grow broader than usual. It seems therefore quite reasonable that in metopical skulls the forehead is broader, this being the natural consequence of the fact that the growth-centrum remains longer in an active state.

With regard to the problem of metopism, observations as well as theoretical considerations have convinced me, that the common opinion about the aetiology of this phenomenon is an erroneous one. As to the facts, I have been unable to confirm the existence of any relation between metopism and a particucular shape of the skull, the frontal suture persisting in dolichocephalic crania as frequently as in brachycephalic ones, and the index cephalicus being in average equal in metopical and nonmetopical skulls. Furthermore, the metopical crania of my collection were not larger than the normal specimens, consequently the average of the brain-weight should be equal in both groups. There is but one fact which I was able to confirm, namely the greater breadth of the forehead in metopical skulls, a phenomenon easily understood as a logical consequence of the protracted activity of the frontal suture.

And as to the theoretical side of the problem, I do not agree with the current opinion that metopism is caused by an increased intracranial pressure, the result of a greater development of the brain. First, because the least indication of such an increased development is wanting, and secondly because in pathological cases, as in hydrocephaly, in which undoubtedly the intracranial pressure had considerably risen, the frontal suture disappears as in normal circumstances.

Before entering into an explanation of my views upon the aetiology of metopism, I wish to discuss briefly the argument that metopism is less frequent in the lower races. As mentioned in the introduction to this paper, this fact is utilized as a proof that metopism, caused by a larger expansion of the brain, should be a symptom of higher intelligence. I think this opinion cannot withstand a serious analysis. If one accepts the principle that metopism is a symptom of intellectual superiority as true, because it is more frequent in culture races, than in uncivilized ones, one must accept also the consequence of this principle, that 
amongst the culture nations those are psychically the most favored in which metopism is the most frequent. Now in the middle region of the continent and in Russia metopism occurs in about 6.5 per cent, according to Schwalbe, Ranke, Gruber and others. In the northern part of Europe, the phenomenon is more frequent, and attains 9.5 per cent according to Bryce, Simon and myself. In Frisians, occupying the northern region of the Netherlands, metopism amounts even to 11.4 per cent. Though the acceptance of the principle should be very flattering for the Dutch people, I do not accept its exactness, metopism having nothing to do with intelligence. I think the interpretation of the different frequency of metopism in the inhabitants of the central and the northern region of the continent to be this: that it is simply a racial difference, the phenomenon occurring more frequently in the Homo nordicus than in the Homo alpinus.

The opinion that the difference in frequency of metopism in the human race is a mere physical anthropological character also holds good with regard to a comparison of civilized and uncivilized races. In the former, metopism is commonly very rare. What may be the reason of it? The authors, who hold that the metopism is the result of an increased intracranial pressure, caused by a somewhat hypernormal growth of the brain, adduce this difference as a proof of the exactness of their doctrine, obviously supposing that such a hypernormal growth does not occur in uncivilized races. In this argument there is a very obvious mistake. Surely the average weight of the brain is a lower one in uncivilized races. But the individual weight of the brain differs in uncivilized races as well as in culture races. Not only among white men, but also among Negroes and Papuans there are individuals with sub-normal, normal and hypernormal volume of their brain. And if really a strongly developed brain should cause an increased pressure upon the. inner surface of the skull, this condition is realized as well in a Papuan with a hypernormal development of his brain, as in an European. Nevertheless in Papuans and Negroes, metopism is rare. I consider this a further proof that the persistence of the frontal suture 
has nothing to do either with brain development, or with the higher or lower degree of intellectual evolution.

Now I wish to express my opinion upon the aetiology of metopism. In the introduction to this paper a brief account is given of the phylogenetic history of the frontal suture, principally in Primates. I summarize that among the Prosimiae in some families the frontal suture, as a rule, persists, while in others, on the contrary, it disappears. In monkeys both frontal bones unite together at a very early stage of development, but in some individuals the suture may persist. In Anthropoids till now the suture has never been seen in an adult specimen. This summary shows that in the course of the phylogenetic evolution of man, originally both frontal bones remained separated; thereupon in the higher degree of evolution the bones coalesced, and finally in man the primitive state presents itself again in a number of individuals. These facts form the basis for a conception of the aetiology of metopism differing from those previously advanced. For it seems to me necessary to begin by discovering the cause which caused the suture to disappear in monkeys. Having elucidated this point, we have approached more closely to the solution of the metopical problem in man. For the possibility must be taken into consideration that the influences which were acting on lower Primates and caused the concrescence of the two frontal bones, have lost their significance and activity in man. If this really happened, it is quite comprehensible that the frontal suture reappears. For in each individual both frontal bones arise separately, the bilateral condition being the rule in the younger stages of development even in such forms in which the individual is born with an already single frontal bone. The metopical suture in an adult individual hence represents no new condition, no alteration of a primitive state, but simply the continuation of an original condition. There must be a special cause for a union of the bones whereas there is no new factor required for the explanation of the fact that they may remain separated. Let us therefore try to find out the primary cause of the concrescence of the frontal bones in monkeys, afterwards we can examine whether this cause became inactive in man or not. 
It is a well established fact that the shape of a bone and especially its internal structure, are the results of the mechanical and muscular forces acting upon it. In accordance with the mechanical principle of securing the maximum of strength with the minimum of material, the cancellous tissues of each bone is so arranged as best to withstand the strains and stresses to which the bone is usually subjected. So the internal architecture of each bone is quite in accordance with the fundamental laws of physics; systems of 'pressure lamellae' running in definite direction are crossed by sets of 'tension lamellae.' A great number of investigators have tried with good results to analyze the structure of the different bones of the human skeleton from this point of view. Only in regard to the skull in general, and particularly the cranial vault, are we without definite knowledge as to the structure of the bony framework of the different bones of the skull and the relation between the statical and dynamical external forces to which it is subjected. The whole of our knowledge is confined to the fact that the structure of the plate-like bones of the cranial vault exhibits the following appearance: the outer and inner surfaces are formed by two compact layers, having sandwiched between them a layer of cancellous tissue.

Nevertheless concerning the cranial vault we find ourselves under relatively favorable circumstances, because the general conditions are so very simple here that the problem can be elucidated sufficiently from a mere theoretical standpoint. For the function of the cranial vault being principally a protective one, the number of mechanical stresses to which the frontal half of the skull is subjected is slight. There are but two factors to take in consideration, namely the weight of the facial cranium with the soft parts of the face as a constant working factor, and the pressure effectuated by the temporal muscle during its contraction. The weight of the facial cranium is transferred surely for the greatest part by means of the zygomatic arches to the middle of the base of the cranium, and so there remains as the only important external force acting upon the anterior and lateral part of the skull, the pressure of the t. mporal muscles, when the jaws are firmly closed. Surely this 
stress will determine the arrangement of the cancellous tissue in the frontal bone. And the variations in the arrangement and the course of the pressure and tension lamellae in different animals, without doubt is caused by the variable relation between the frontal bone and the Musculus temporalis. If the muscle arises largely from the frontal bone the internal structure of the anterior region of the cranial vault will be largely influenced by the same. It is obvious that in such a case the frontal and sagittal suture are primarily subject to this influence, as their course is perpendicular to that of the fibers of the muscle.

I think this idea is sufficient to demonstrate why in lower Primates the frontal suture persists, while in the higher Primates it regularly disappears. For the stress of the masticatory muscles tends to compress the skull in a transverse direction and the vault of the skull will withstand this force by a system of trajectories, running on a frontal plane. Now it is not difficult to understand that it is of advantage that the trajectories do not meet with an open suture in their course. And so the fate of the metopical suture in Primates will depend upon the topographical relation between the temporal muscle and the frontal bone. If the muscle arises from the frontal bones a system of pressure and tension lamallae will be developed in it crossing the median line and hence necessitating the union of the two primary frontal bones. If on the contrary, the bone remains free from the dynamical influence of the muscle, there is no reason for the union of the two bones.

In figure 1 an attempt is made to elucidate the above described idea by means of a very simple scheme. It represents a frontal section of the anterior part of the vault of the skull, with the temporal muscle on both sides. The direction in which the vault will be narrowed by the stress of the contracting muscle is indicated by two arrows. It is obvious that in order to withstand this stress pressure trajectories will be developed in the vertical parts of the vault, under the direct influence of this force. The compression in the indicated direction will produce a tension in the top of the vault. And while in the vertical parts of it the cancellous tissue will arrange itself in pressure 
lamellae, on the top a system of tension lamellae will arise. In the figure both systems are represented by some simple lines. I admit it is a purely theoretical construction, which I have tried, however, to bring in accordance with the principles of mechanics. The point upon which I will lay some stress, is that the tension lamellae necessarily must cross the median plane. And because an interruption in their course by a suture would be contrary to their mechanical function, the two frontal bones unite together. Now we will examine in how far the anatomical conditions in the different Primates agree with the principles worked out above.

It is needless to give a long description of the anatomical conditions in several specimens, for the inspection of some few

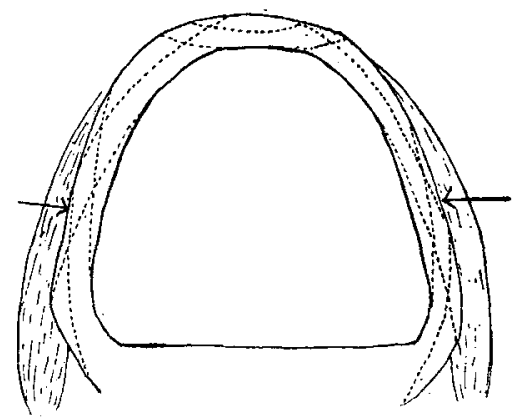

crania suggests the regularity in the special groups of the Primates. I will confine myself therefore to treat each group as a whole.

The examination of the prosimian skull shows that in this lowest group of Primates the frontal suture is a constant element in the system of sutures, disappearing nearly at the same time as the other sutures. I regret to have at my disposal only a small number of skulls of prosimiae. Hence it is impossible for me to give a summary of the age at which the metopical suture disappears in the different genera of this group of Primates. The small number of skulls in my possession indicate that a considerable variability exists as to this point in the different genera of the Prosimiae. So I found among five adult skulls of Lemur only one specimen with the system of sutures still wholly 
intact, including the metopical suture. In the others the system had completely disappeared. In three adult crania of Avahis on the contrary, apparently of old individuals, all sutures including the metopical, were still present, and so it was in two old crania of Nycticebus. It thus seems that the sutures in Prosimiae close at a very different stage in the different genera of this family. But for the present it suffices to know that the disappearance of the metopical suture takes place simultaneously with that of the other elements of the system. There is no special factor necessitating the same to close at an earlier period than the other sutures. In this respect the Prosimiae differ from the monkeys and apes in which the closure of the frontal suture always precedes those of the other sutures, and often very considerably. From this we may conclude that the influence compelling the metopical suture in monkeys and apes to disappear, is absent in Prosimiae. Now a comparison of the topographical relations between the temporal muscle and the frontal bone in the lower and higher Primates, reveals that in Prosimiae the muscle does not arise from the frontal bone at all. The reason for this is obvious. In Prosimiae the lateral wall of the orbit is a very incomplete one, and frequently also the floor of this fossa is restricted to a foremost part. As a rule the outer wall only is represented by an arch extending from the facial root of the zygomatic arch to the parietal margin of the frontal bone. This insertion of the orbital arch at the hindermost border of the frontal bone causes the latter to be situated completely in front of the temporal fossa, hence the temporal muscle cannot extend its origin forward upon the frontal bone. In monkeys, as in apes and man, the outer wall of the orbit is a complete one, formed partly by the orbital surfaces of the zygomatic bone and the great wing of the sphenoid bone. By this outer wall the orbit is separated almost completely from the temporal fossa and the plane of entrance of the orbit is considerably turned: In Prosimiae the inclination of the latter is more a lateral than a frontal one, the axis of the orbit making a more open angle with the median plane. But in monkeys the plane of entrance is turned, being directed principally forward and but slightly out- 
ward. The axis of the orbital fossa is making therefore a more acute angle with the median plane. In consequence of the rotation of the plane of entrance of the orbit, the insertion of the primitive orbital arch at the frontal bone was shifted from the hindermost border of the bone forward, so that a part of the outer surface of the frontal bone is added to the temporal fossa. By this enlargement of the fossa the temporal muscle was enabled to arise to a smaller or greater extent from the frontal bone.

The differences between Prosimiae and the higher Primates are clearly shown by the figures 2 to 9 . In these figures the lateral and superior view of some prosimian and simian skulls is drawn. The course of the main sutures and also the extension of the temporal muscle is indicated. Figures 2 and 3 represent lateral views of the cranium of Avahis sinavensis and of Stenops gracilis respectively. In both it is obvious that the frontal bone is completely excluded from the temporal fossa, and that there are no fibers of the temporal muscle arising from this bone. Hence it is easy to understand that in those crania the frontal suture persists, as is shown in figure 4, representing the superior view of the skull of an Avahis niger. The frontal bone remains free from the dynamical influence of the temporal muscle, its anatomical significance is a restricted one. It functions only as roof of the orbits and the foremost narrow part of the cavity of the skull. In consequence of the absence of forces acting upon this bone, its system of trajectories cannot be strongly developed. Hence there is no reason for both frontal bones to unite.

Quite the contrary happens in the skulls of monkeys from the Old and New World, as is illustrated by figures $5,6,7$, and 8 . Figure 5 represents a side view and figure 6 a superior view of the skull of Chrysothrix, a platyrrhinic monkey, figure 7 a side $\mathrm{v}$ ew of the skull of Macacus, and figure 8 such a one of a female Gorilla. The extension of the temporal muscle and the course of the sutures in the cranial vault are drawn. These figures require but little comment. In all it is clear that the frontal bone participates in the formation of the temporal fossa, and that no small part of the temporal muscle takes origin from this bone. 

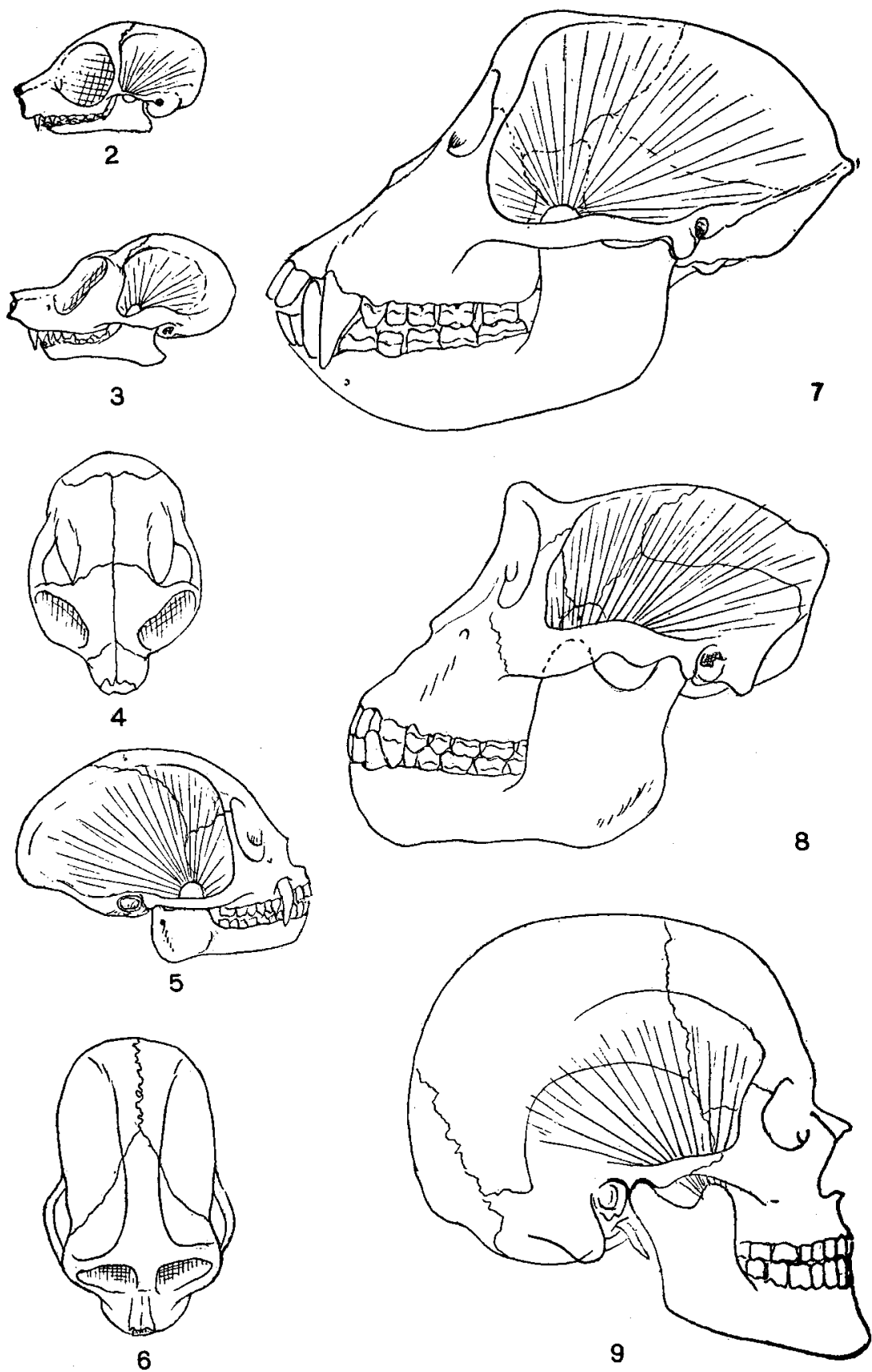
In Macacus and Gorilla the origin of the muscle reaches to the median line, so that there is but a small triangular part of the outer surface of the bone uncovered by the muscle, while in Chrysothrix a narrow strip on both sides of the median line remains free from the origin of the muscle. It requires no special argument to show that the forces executed by the contracting muscle upon the frontal bone must give rise to a system of trajectories in it, able to withstand the strains on its outer surface. And it is important to draw attention to the fact that the fibers of the muscle are directed perpendicularly to the median line and consequently also with regard to the frontal suture, the forehead being directed horizontally immediately behind the superciliary arch. This condition surely favors the formation of trajectories crossing the median line and causing the frontal suture to disappear, as really occurs in all monkeys and apes. In man the condition is greatly changed, though a small part of the frontal bone is still participating in the formation of the temporal fossa, as shown in figure 9 . There are two circumstances by which the relation between the temporal muscle and the frontal bone became altered from that obtaining in monkeys. Firstly, the frontal bone in man is much larger, and the surface of it occupied by the origin of the temporal muscle is considerably smaller in man than in apes. The pressure of the muscle upon the outer face of this bone in man cannot be a very strong one, hence its influence upon the inner structure surely is of little importance. In this respect the condition in man is getting closer to that in Prosimiae.

The second circumstance peculiar to man is the well-pronounced curve of his frontal bone. By this curve the greater part of this bone rises vertically above the orbits. In apes, as pointed out, the fibers of the temporal muscle are directed perpendicularly to the whole length of the frontal suture. In man this condition is altered, for in consequence of his strongly curved forehead the greater part of the frontal suture is situated in front of the anterior border of the temporal muscle, and moreover is directed nearly parallel to this border. 
By these two circumstances the frontal suture in man becomes independent from the dynamical influence of the temporal muscle. Hence in man there is a return to the conditions as met with in Prosimians, though the anatomy of the skull and the muscles is quite different. The mechanical cause for the disappearance of the suture in monkeys having fallen out, the circumstances become very favorable to the persistence. Now it is obvious that these conditions act most favorably in individuals with a more prominent forehead and a less pronounced development of the masticatory musculature. In the white race therefore, the possibility for the persistance of the suture is far greater than in the races with a more flattened forehead, a higher development of the dentition and of the temporal muscle. And this may be considered the cause, accounting for the fact that commonly metopism is more frequent in Europeans than in Negroes or Australians. 